\title{
Aplicabilidade de equações na avaliação da composição corporal da população brasileira
}

\author{
Applicability of equations in assessing the body \\ composition of the Brazilian population
}

Fabiane Aparecida Canaan REZENDE ${ }^{1}$

Lina Enriqueta Frandsen Paez Lima ROSADO²

Silvia Eloiza PRIORE²

Sylvia do Carmo Castro FRANCESCHINI²

\section{RE S U M O}

O excesso de gordura corporal está, freqüentemente, associado a alterações metabólicas importantes e às doenças crônicas, como diabetes, hipertensão arterial e dislipidemias. A disponibilidade de métodos que permitam estimar a composição corporal é fundamental para avaliação de atletas e do estado de saúde de indivíduos. Entre os métodos existentes, as medidas de dobras cutâneas são habitualmente utilizadas na população brasileira, devido à sua facilidade e relativa precisão em estimar a gordura corporal. Entretanto, existem limitações quanto à utilização desse método, que podem implicar em erros de estimativas da gordura corporal importantes. A proposta deste trabalho é descrever os estudos que validaram essas equações e os principais fatores que influenciam a acurácia das mesmas na população em geral. Também são discutidos aspectos quanto à aplicabilidade dessas equações na população brasileira.

Termos de indexação: composição corporal; dobras cutâneas; validade.

\section{A B S T R A C T}

An excess of body fat is frequently associated with important metabolic alterations and chronic diseases, such as diabetes, hypertension and dyslipidemia. The availability of methods to assess body composition is essential to evaluate athletes and the health status of individuals. Among the existent methods, the skinfold measurement is broadly used among the Brazilian population, due to its ease-of-use and relative precision to estimate body fat. However, there are some limitations related to the use of this method as it may result is gross mistakes when estimating body fat. The aim of this review is to describe the studies that validated those equations and the main factors that have influenced their accuracy in the general population. Some aspects related to the application of those equations in the Brazilian population are also discussed.

Indexing terms: body composition; skinfold thickness; validity.

\footnotetext{
1 Mestranda em Ciência da Nutrição, Departamento de Nutrição e Saúde, Universidade Federal de Viçosa. Viçosa, MG, Brasil.

2 Departamento de Nutrição e Saúde, Universidade Federal de Viçosa. Av. P.H. Rolfs, s/n, 36571-000, Campus Universitário.

Viçosa, MG, Brasil. Correspondência para/Correspondence to: S.C.C. FRANCESCHINI.
} 


\section{N T R O D U Ç Ã O}

A determinação da composição corporal tem grande importância na prática clínica e na avaliação de populações, devido, principalmente, à associação da gordura corporal com diversas alterações metabólicas. Vários estudos demonstram que a quantidade de tecido adiposo e sua distribuição estão associadas a elevados valores de pressão arterial, dislipidemias, com concentrações elevadas de triglicerídeos e reduzidas de colesterol de alta densidae (HDL), intolerância à glicose e resistência insulínica, os quais contribuem para a elevação do risco cardiovascular ${ }^{1,2}$.

Diante da influência da quantidade de gordura corporal no estado de saúde dos indivíduos, são necessários métodos capazes de avaliar, de forma precisa e confiável, a quantidade de gordura corporal em relação à massa corporal total.

Há uma variedade de técnicas para a avaliação da composição corporal, como a tomografia computadorizada, ressonância magnética, impedância bioelétrica, dobras cutâneas, medida de absorção do raios $X$ de dupla energia (DEXA) e pesagem hidrostática, sendo estes dois últimos considerados como "padrões-ouro" nos estudos de validação ${ }^{3}$. A escolha do método a ser utilizado dependerá de quais compartimentos corporais se pretende determinar e de aspectos como, custo, validade, aplicabilidade do método e grau de treinamento necessário ao avaliador ${ }^{4}$.

Testes estatísticos, como correlação linear de Pearson e Teste " $t$ " de student, são utilizados para verificar a validade dos métodos. Além disso, a capacidade do avaliador de reproduzir as medidas, a padronização das técnicas e a utilização de instrumentos calibrados e precisos são fatores determinantes para a obtenção de dados confiáveis ${ }^{3}$.

Entre os métodos antropométricos, as dobras cutâneas são habitualmente utilizadas para determinação do percentual de gordura corporal, devido ao baixo custo operacional e à relativa simplicidade de utilização³.
Existem, na literatura, mais de 100 equações que utilizam as medidas de dobras cutâneas e outras medidas antropométricas, como circunferências, para determinação da composição corporal $^{4}$. Entretanto, a validade de equações que utilizam medidas de dobras cutâneas para predizer a composição corporal é restrita para a população da qual essas equações foram derivadas. Portanto, a validade e acurácia dessas equações precisam ser cuidadosamente avaliadas no momento da sua escolha ${ }^{5}$.

Para selecionar o método e a equação mais adequados, fatores como, idade, sexo, etnia, nível de atividade física e quantidade de gordura corporal, precisam ser levados em consideração. O ideal é que as características da população que se quer avaliar sejam similares às da amostra utilizada no processo de validação da equação escolhida ${ }^{6}$.

Esse trabalho se propõe a descrever os artigos de validação das equações mais utilizadas no Brasil: Durnin \& Wormersley ${ }^{7}$, Jackson \& Pollock ${ }^{8}$ e Jackson et al. ${ }^{9}$, e discutir aspectos relacionados à validade e aplicabilidade dessas equações para a população brasileira.

\section{Durnin \& Wormersley}

Em 1967, Durnin \& Rahaman conduziram um estudo na Inglaterra com o objetivo de formular equações simples para predizer a quantidade de gordura corporal. Participaram da pesquisa, indivíduos com idade entre 12 e 34 anos, sendo 105 adultos jovens e 86 adolescentes. Medidas antropométricas, de dobras cutâneas e de densidade corporal por pesagem hidrostática foram obtidas para a elaboração das equações ${ }^{10}$.

Os resultados confirmaram diferentes percentuais de gordura corporal segundo o sexo, sendo que mulheres, independentemente da faixa etária, apresentaram valores percentuais mais elevados, quando comparadas aos homens. Os coeficientes de correlação entre as medidas de dobras cutâneas e de densidade corporal variaram de $0,760-0,835^{10}$. 
A partir desses resultados, em 1974, Durnin \& Wormersley ${ }^{7}$ examinaram novamente a relação entre medidas de dobras cutâneas e de densidade corporal em indivíduos de diferentes faixas etárias, e determinaram a acurácia com a qual a densidade corporal poderia ser estimada por medidas de dobras cutâneas.

A amostra do estudo foi composta por 209 homens e 272 mulheres, com idade entre 16 e 72 anos, sendo que os indivíduos eram, em sua maioria, sedentários e pertencentes à classe média7. Foram aferidas medidas de dobras cutâneas (bicipital, tricipital, subescapular e supra-ilíaca) e circunferências (do braço, da coxa e da panturrilha). O percentual de gordura corporal foi calculado a partir da densidade corporal obtida por pesagem hidrostática ${ }^{7}$.

Os coeficientes de correlação entre a soma de duas ou mais dobras cutâneas (na transformação logarítmica) e a densidade corporal, variaram entre 0,7 e 0,9 para os diferentes grupos de idade de ambos os sexos ${ }^{7}$. O que foi observado com esse resultado, em relação ao primeiro estudo realizado por Durnin \& $\operatorname{Rahaman}^{10}$, é que a correlação obtida nesta amostra apresentou maior variação, justificada pela grande amplitude da faixa etária.

Utilizando a transformação logarítmica da soma de quatro dobras cutâneas, o erro padrão da estimativa da densidade corporal foi de $0,0084 \mathrm{~kg} / \mathrm{m}^{3}(0,0073-0,0092)$ para homens e $0,0102 \mathrm{~kg} / \mathrm{m}^{3}(0,0082-0,0125)$ para as mulheres, valores menores do que aqueles encontrados com o valor absoluto das medidas no sexo masculino, enquanto nenhuma diferença consistente foi encontrada no sexo feminino. A incorporação das outras medidas, junto às dobras cutâneas para a elaboração de equações mais complexas, resultou em um aumento mínimo da acurácia ${ }^{7}$.

A densidade corporal apresentou diferença significante entre os grupos de idade, sendo que o aumento da idade foi acompanhado por uma redução da densidade corporal em ambos os sexos. Essa diminuição foi ligeiramente maior nos homens, com uma redução de $0,005 \times 10^{3} \mathrm{~kg} / \mathrm{m}^{3}$ por década, sendo mais observada em indivíduos obesos $^{7}$.

Os autores discutem que essa diminuição da densidade da massa livre de gordura ocorreria devido a mudanças no compartimento ósseo, e que a diminuição da gordura subcutânea com a idade estaria ocorrendo devido a uma maior localização interna do tecido adiposo. Além disso, discutem que a compressibilidade da dobra cutânea pode tornar-se maior em indivíduos mais velhos, levando a uma estimativa menor do percentual de gordura corporal ${ }^{7}$.

Ao final das análises foi gerada uma tabela contendo ampla faixa do somatório das quatro dobras com o equivalente percentual de gordura corporal, de acordo com idade e sexo (Tabela 1) ${ }^{7}$.

\section{Jackson \& Pollock ${ }^{8}$}

\section{Equações generalizadas para predizer densidade corporal em homens}

Neste estudo, os autores buscaram derivar equações de regressão generalizadas que pudessem fornecer estimativas de densidade corporal para homens, variando em idade e composição corporal. Maior atenção foi dada à relação curvilínea entre as medidas de dobras cutâneas e de densidade corporal, e também à influência da idade sobre as mesmas ${ }^{8}$.

Foram incluídos 403 homens adultos com idade entre 18 e 61 anos, os quais variaram, consideravelmente, na estrutura corporal, composição corporal e prática de atividade física. A amostra total foi aleatoriamente dividida em amostra de regressão $(n=308)$ e de validação $(n=95)$. Não houve diferenças significantes entre as características das duas amostras ${ }^{8}$.

Foram obtidas as medidas de peso, estatura, circunferências (do antebraço e da cintura) e dobras cutâneas (torácica, axilar, tricipital, subescapular, abdominal, supra-ilíaca e da coxa). O compasso modelo Lange foi utilizado para a medição das dobras cutâneas. A densidade 
corporal foi determinada pelo método de pesagem hidrostática, para posterior cálculo do percentual de gordura corporal ${ }^{3}$.

As análises desse estudo incluíram a soma de sete e de três dobras cutâneas (torácica, abdominal e da coxa). O método de transformação logarítmica também foi aplicado para a soma dessas medidas e usado na comparação com o trabalho de Durnin \& Wormersley ${ }^{7,8}$.

Nesse estudo, idade e composição corporal variaram amplamente, com elevados valores de desvio-padrão, mostrando que existiam diferenças consideráveis entre os homens de ambas as amostras ${ }^{8}$.

Tabela 1. Percentual de gordura corporal de acordo com a soma de quatro dobras cutâneas bíceps, tríceps, subescapular, supra-ilíaca) de homens e mulheres de diferentes idades.

\begin{tabular}{|c|c|c|c|c|c|c|c|c|}
\hline \multirow{2}{*}{ Dobras Cutâneas } & \multicolumn{4}{|c|}{ Homens (idade em anos) } & \multicolumn{4}{|c|}{ Mulheres (idade em anos) } \\
\hline & $17-29$ & $30-39$ & $40-49$ & $\geq 50$ & $16-29$ & $30-39$ & $40-49$ & $\geq 50$ \\
\hline 15 & 4,8 & - & - & - & 10,5 & - & - & - \\
\hline 20 & 8,1 & 12,2 & 12,2 & 12,6 & 14,1 & 17,0 & 19,8 & 21,4 \\
\hline 25 & 10,5 & 14,2 & 15,0 & 15,6 & 16,8 & 19,4 & 22,2 & 24,0 \\
\hline 30 & 12,9 & 16,2 & 17,7 & 18,6 & 19,5 & 21,8 & 24,5 & 26,6 \\
\hline 35 & 14,7 & 17,7 & 19,6 & 20,8 & 21,5 & 23,7 & 26,4 & 28,5 \\
\hline 40 & 16,4 & 19,2 & 21,4 & 22,9 & 23,4 & 25,5 & 28,2 & 30,3 \\
\hline 45 & 17,7 & 20,4 & 23,0 & 24,7 & 25,0 & 26,9 & 29,6 & 31,9 \\
\hline 50 & 19,0 & 21,5 & 24,6 & 26,5 & 26,5 & 28,2 & 31,0 & 33,4 \\
\hline 55 & 20,1 & 22,5 & 25,9 & 27,9 & 27,8 & 29,4 & 32,1 & 34,6 \\
\hline 60 & 21,2 & 23,5 & 27,1 & 29,2 & 29,1 & 30,6 & 33,2 & 35,7 \\
\hline 65 & 22,2 & 24,3 & 28,2 & 30,4 & 30,2 & 31,6 & 34,1 & 36,7 \\
\hline 70 & 23,1 & 25,1 & 29,3 & 31,6 & 31,2 & 32,5 & 35,0 & 37,7 \\
\hline 75 & 24,0 & 25,9 & 30,3 & 32,7 & 32,2 & 33,4 & 35,9 & 38,7 \\
\hline 80 & 24,8 & 26,6 & 31,2 & 33,8 & 33,1 & 34,3 & 36,7 & 39,6 \\
\hline 85 & 25,5 & 27,2 & 32,1 & 34,8 & 34,0 & 35,1 & 37,5 & 40,4 \\
\hline 90 & 26,2 & 27,8 & 33,0 & 35,8 & 35,6 & 35,8 & 38,3 & 41,2 \\
\hline 95 & 26,9 & 28,4 & 33,7 & 36,6 & 36,4 & 36,5 & 39,0 & 41,9 \\
\hline 100 & 27,6 & 29,0 & 34,4 & 37,4 & 37,1 & 37,2 & 39,7 & 42,6 \\
\hline 105 & 28,2 & 29,6 & 35,1 & 38,2 & 37,8 & 37,9 & 40,4 & 43,3 \\
\hline 110 & 28,8 & 30,1 & 35,8 & 39,0 & 38,4 & 38,6 & 41,0 & 43,9 \\
\hline 115 & 29,4 & 30,6 & 36,4 & 39,7 & 39,0 & 39,1 & 41,5 & 44,5 \\
\hline 120 & 30,0 & 31,1 & 37,0 & 40,4 & 39,6 & 39,6 & 42,0 & 45,1 \\
\hline 125 & 30,5 & 31,5 & 37,6 & 41,1 & 40,2 & 40,1 & 42,5 & 45,7 \\
\hline 130 & 31,0 & 31,9 & 38,2 & 41,8 & 40,8 & 40,6 & 43,0 & 46,2 \\
\hline 135 & 31,5 & 32,3 & 32,7 & 42,4 & 41,3 & 41,1 & 43,5 & 46,7 \\
\hline 140 & 32,0 & 32,7 & 39,2 & 43,0 & 41,8 & 41,6 & 44,0 & 47,2 \\
\hline 145 & 32,5 & 33,1 & 39,7 & 43,6 & 42,3 & 42,1 & 44,5 & 47,7 \\
\hline 150 & 32,9 & 33,5 & 40,2 & 44,1 & 42,8 & 42,6 & 45,0 & 48,2 \\
\hline 155 & 33,3 & 33,9 & 40,7 & 44,6 & 43,3 & 43,1 & 45,4 & 48,7 \\
\hline 160 & 33,7 & 34,3 & 41,2 & 45,1 & 43,7 & 43,6 & 45,8 & 49,2 \\
\hline 165 & 34,1 & 34,6 & 41,6 & 45,6 & 44,1 & 44,0 & 46,2 & 49,6 \\
\hline 170 & 34,5 & 34,8 & 42,0 & 46,1 & - & 44,4 & 46,6 & 50,0 \\
\hline 175 & 34,9 & - & - & - & - & 44,8 & 47,0 & 50,4 \\
\hline 180 & 35,3 & - & - & - & - & 45,2 & 47,4 & 50,8 \\
\hline 185 & 35,6 & - & - & - & - & 45,6 & 47,8 & 51,2 \\
\hline 190 & 35,9 & - & - & - & - & 45,9 & 48,2 & 51,6 \\
\hline 195 & - & - & - & - & - & 46,2 & 48,5 & 52,0 \\
\hline 200 & - & - & - & - & - & 46,5 & 48,8 & 52,4 \\
\hline 205 & - & - & - & - & - & - & 49,1 & 52,7 \\
\hline 210 & - & - & - & - & - & - & 49,4 & 53,0 \\
\hline
\end{tabular}

Fonte: Durnin \& Wormersley ${ }^{7}$ 
A correlação entre a soma de três e sete dobras cutâneas foi alta $(r=0,98)$, demonstrando que as dobras cutâneas torácica, abdominal e da coxa podem ser utilizadas na avaliação desses indivíduos, devido à maior facilidade e rapidez na obtenção dessas medidas 8 .

Foram derivadas oito equações que diferiram quanto ao número de dobras cutâneas, somadas ao modelo matemático utilizado e à inclusão da circunferência da cintura e do antebraço. A idade foi levada em consideração para todas as equações (Tabela 2 ) $^{8}$.

Após a derivação das equações, as mesmas foram validadas em uma segunda amostra, e as análises mostraram que essas equações foram capazes de predizer com acurácia a densidade corporal em diferentes idades e a quantidade de gordura corporal ${ }^{8}$.

\section{Jackson \& Pollock $^{9}$}

\section{Equações generalizadas para predizer densidade corporal em mulheres}

A proposta desse estudo foi derivar equações de regressão generalizadas que pudessem fornecer estimativas da densidade corporal com erro mínimo para mulheres, variando em idade e composição corporal. A relação curvilínea entre as medidas de dobras cutâneas e a densidade corporal foi investigada, assim como a influência da idade sobre a densidade corporal ${ }^{9}$.

Foram avaliadas 331 mulheres entre 18 e 55 anos, que apresentaram considerável variabilidade quanto à estrutura e composição corporal e à prática de exercícios. Essa amostra foi dividida aleatoriamente em amostra de regressão (249 mulheres), para derivar as equações generalizadas, e amostra de validação (82 mulheres), para verificar a acurácia das equações geradas ${ }^{9}$.

A avaliação antropométrica incluiu as medidas de peso, estatura, circunferência do quadril e sete dobras cutâneas (tricipital, axilar, subescapular, abdominal, supra-ilíaca, torácica e da coxa). O compasso modelo Lange foi utilizado para a medição das dobras cutâneas. A pesagem hidrostática foi utilizada para determinar a densidade corporal e, a partir daí, calculou-se o percentual de gordura corporal ${ }^{9}$.

As equações foram derivadas utilizando a soma de sete, quatro (tricipital, supra-ilíaca, abdominal e da coxa) e três dobras cutâneas

Tabela 2. Equações de regressão generalizadas para predição da densidade corporal (DC) de homens adultos com idade entre 18 e 61 anos.

\begin{tabular}{|c|c|c|c|}
\hline Número & Equação de regressão & r & EP \\
\hline \multicolumn{4}{|c|}{ Soma de sete dobras cutâneas } \\
\hline 1 & $D C=1,11200000-0,00043499\left(X_{1}\right)+0,00000055\left(X_{1}\right)^{2}-0,00028826\left(X_{3}\right)$ & 0,902 & 0,0078 \\
\hline 2 & $\begin{array}{l}D C=1,10100000-0,00041150\left(X_{1}\right)+0,00000069\left(X_{1}\right)^{2}-0,00022631\left(X_{3}\right)-0,0059239 \\
\left(X_{4}\right)+0,0190632\left(X_{5}\right)\end{array}$ & 0,916 & 0,0073 \\
\hline 3 & $D C=1,21394-0,03101\left(\log X_{1}\right)-0,00029\left(X_{3}\right)$ & 0,893 & 0,0082 \\
\hline 4 & $D C=1,17615-0,02394\left(\log X_{1}\right)-0,00029\left(X_{3}\right)-0,0070(X 4)+0,02120\left(X_{5}\right)$ & 0,917 & 0,0073 \\
\hline \multicolumn{4}{|c|}{ Soma de três dobras cutâneas } \\
\hline 5 & $D C=1,1093800-0,0008267\left(X_{2}\right)+0,0000016\left(X_{2}^{12}-0,0002574\left(X_{3}\right)\right.$ & 0,905 & 0,007 \\
\hline 6 & $\begin{array}{l}D C=1,0990750-0,0008209\left(X_{2}\right)+0,0000026\left(X_{2}\right)^{2}-0,0002017\left(X_{3}\right)-0,005675\left(X_{4}\right)+ \\
0,018586\left(X_{5}\right)\end{array}$ & 0,918 & 0,0072 \\
\hline 7 & $D C=1,18860-0,03049\left(\log X_{2}\right)-0,00027\left(X_{3}\right)$ & 0,888 & 0,0083 \\
\hline 8 & $D C=1,15737-0,02288\left(\log X_{2}\right)-0,00019\left(X_{3}\right)-0,0075(X 4)+0,0223\left(X_{5}\right)$ & 0,915 & 0,0073 \\
\hline
\end{tabular}

Fonte: Jackson \& Pollock ${ }^{8} ; X_{1}=$ Soma das dobras cutâneas: torácica, axilar, tricipital, subescapular, abdominal, supra-ilíaca, coxa frontal; $X_{2}=$ Soma das dobras cutâneas da coxa, tórax e abdômen; $X_{3}=$ Idade; $X_{4}=$ Circunferência da cintura; $X_{5}=$ Circunferência do antebraço; $r=$ Coeficiente de correlação; EP= Erro-padrão. 
(tricipital, suprailíaca e da coxa) por intermédio de análises de regressão múltipla. Foram utilizadas a forma quadrática e a transformação logarítmica, para comparação com as equações de Durnin \& Wormersley ${ }^{7}$. A soma de quatro e três dobras foi utilizada devido à maior facilidade em trabalho de campo?.

As médias de dobras cutâneas, densidade corporal e percentual de gordura, na amostra de validação, exibiram uma tendência não linear por grupo de idade (17-19, 20-29, 30-39, 40-49, 50-59 anos) e nenhuma diferença significativa foi encontrada entre as faixas etárias 9 .

Foram geradas 18 equações que diferiram quanto ao número de dobras cutâneas, ao modelo matemático utilizado e quanto à inclusão das variáveis idade e circunferência do quadril (Tabela 3) .

Os coeficientes de correlação entre a soma de sete, de quatro e de três dobras na amostra de regressão foram altos $(r>0,966)$, entretanto, as correlações dessas medidas com os valores de densidade corporal variaram entre 0,838 e 0,867, com erro-padrão entre 3,6\% e 4,0\% de gordura corporal. Nessa amostra de validação as correlações entre densidade corporal, obtida por pesagem hidrostática, e por dobras cutâneas foram menores $(0,799-0,827)$, com erro-padrão entre $3,7 \%$ e $4,0 \%$ de gordura corporal ${ }^{9}$.

Os valores de erro-padrão foram maiores para as categorias de idade acima de 40 anos e percentual de gordura corporal abaixo de $20 \%$, evidenciando uma acurácia menor dessas equações para predizer a gordura corporal em grupos com essas características. A transformação logarítmica produziu valores de erros-padrão ainda mais elevados ${ }^{9}$.

\section{Fatores Determinantes da Acurácia das Equações}

A aplicabilidade dessas fórmulas de maneira generalizada vem sendo bastante

Tabela 3. Equações de regressão generalizadas para predição de densidade corporal de mulheres adultas de 18 a 55 anos.

\begin{tabular}{|c|c|c|c|}
\hline Número & Equação de regressão & r & EP \\
\hline \multicolumn{4}{|c|}{ Soma de sete dobras cutâneas } \\
\hline 1 & $D C=1,0970-0,00046971\left(X_{1}\right)+0,00000056\left(X_{1}\right)^{2}-0,00012828\left(X_{4}\right)$ & 0,852 & 0,0083 \\
\hline 2 & $D C=1,23173-0,03841\left(\log X_{1}\right)-0,00015\left(X_{4}\right)$ & 0,850 & 0,0084 \\
\hline 3 & $D C=1,1470-0,00042359\left(X_{1}\right)+0,00000061\left(X_{1}\right)^{2}-0,00065200\left(X_{5}\right)$ & 0,865 & 0,0080 \\
\hline 4 & $D C=1,25475-0,03100\left(\log X_{1}\right)-0,00068\left(X_{5}\right)$ & 0,864 & 0,0080 \\
\hline 5 & $D C=1,1470-0,00042930\left(X_{1}\right)+0,00000065\left(X_{1}\right)^{2}-0,00009975\left(X_{4}\right)-0,00062415(X 5)$ & 0,867 & 0,0079 \\
\hline 6 & $D C=1,25186-0,03048\left(\log X_{1}\right)-0,00011\left(X_{4}\right)-0,00064\left(X_{5}\right)$ & 0,867 & 0,0079 \\
\hline \multicolumn{4}{|c|}{ Soma de sete dobras cutâneas } \\
\hline 7 & $D C=1,0960950-0,0006952\left(X_{2}\right)+0,0000011\left(X_{2}\right)^{2}-0,0000714\left(X_{4}\right)$ & 0,849 & 0,0084 \\
\hline 8 & $D C=1,21993-0,03936\left(\log X_{2}\right)-0,00011\left(X_{4}\right)$ & 0,845 & 0,0085 \\
\hline 9 & $D C=1,1443913-0,0006523\left(X_{2}\right)+0,0000014\left(X_{2}\right)^{2}-0,0006053\left(X_{5}\right)$ & 0,861 & 0,0081 \\
\hline 10 & $D C=1,24374-0,03162\left(\log X_{2}\right)-0,00066\left(X_{5}\right)$ & 0,859 & 0,0081 \\
\hline 11 & $D C=1,1454464-0,0006558\left(X_{2}\right)+0,0000015\left(X_{2}\right)^{2}-0,0000604\left(X_{4}\right)-0,0005981\left(X_{5}\right)$ & 0,862 & 0,0081 \\
\hline 12 & $D C=1,241721-0,031069\left(\log X_{2}\right)-0,000077\left(X_{4}\right)-0,000635\left(X_{5}\right)$ & 0,861 & 0,0081 \\
\hline \multicolumn{4}{|c|}{ Soma de sete dobras cutâneas } \\
\hline 13 & $D C=1,0994921-0,0009929\left(X_{3}\right)+0,0000023\left(X_{3}\right)^{2}-0,0001395\left(X_{4}\right)$ & 0,842 & 0,0086 \\
\hline 14 & $D C=1,21389-0,04057\left(\log X_{3}\right)-0,00016\left(X_{4}\right)$ & 0,838 & 0,0087 \\
\hline 15 & $D C=1,1466399-0,0009300\left(X_{3}\right)+0,0000028\left(X_{3}\right)^{2}-0,0006171\left(X_{5}\right)$ & 0,851 & 0,0084 \\
\hline 16 & $D C=1,23824-0,03248\left(\log X_{3}\right)-0,00067\left(X_{5}\right)$ & 0,849 & 0,0084 \\
\hline 17 & $D c=1,1470292-0,0009376\left(X_{3}\right)+0,0000030\left(X_{3}\right)^{2}-0,0001156\left(X_{4}\right)-0,0005839\left(X_{5}\right)$ & 0,854 & 0,0083 \\
\hline 18 & $D C=1,23530-0,03192\left(\log X_{3}\right)-0,00013\left(X_{4}\right)-0,00062\left(X_{5}\right)$ & 0,853 & 0,0083 \\
\hline
\end{tabular}

Fonte: Jackson et al. $9 ; X_{1}=$ Soma de sete dobras cutâneas; $X_{2}=$ Soma das dobras cutâneas do tríceps, abdômen, coxa e supra-ilíaca; $X_{3}=$ Soma das dobras cutâneas do tríceps, coxa e supra-ilíaca; $X_{4}=$ Idade; $X_{5}=$ Circunferência do quadril; $r=$ Coeficiente de correlação; $E P=$ Erro-padrão. 
questionada, e vários estudos têm avaliado a acurácia dessas equações em predizer a gordura corporal em diferentes populações. Os resultados têm mostrado a inadequação dessas fórmulas em vários grupos populacionais, considerando que aspectos como sexo, etnia, idade, quantidade de gordura corporal e nível de atividade física, influenciam de maneira importante a capacidade preditiva dessas equações. Alguns desses fatores serão descritos a seguir.

De maneira geral, as diferenças quanto à composição corporal entre os sexos compreendem elevada quantidade de massa magra em homens e maiores percentuais de gordura corporal em mulheres. Esse padrão pode apresentar-se alterado, de acordo com os níveis de atividade física praticados pelos indivíduos. Essa diferença de composição corporal entre os gêneros já está bem estabelecida e sempre deve ser considerada em estudos em que a composição corporal é avaliada $^{11,12}$.

A idade é um dos fatores determinantes para as alterações da composição corporal. Com o avançar da idade, ocorrem mudanças na mineralização óssea e hidratação da massa magra, além de redistribuição e aumento da gordura corporal, com redução da gordura corporal periférica e subcutânea e acúmulo intra-abdominal ${ }^{13}$.

Estudos compreendendo amostras representativas com ampla faixa de idade mostram que ocorre um aumento progressivo da gordura corporal com o aumento da idade e uma redução da massa magra e da gordura corporal em torno de 50 a 60 anos, dependendo do sexo 11,12,14.

Diante dos dados existentes na literatura, percebe-se que equações muito generalizadas, que compreendem amplas faixas etárias, devem ser utilizadas com cautela. A inclusão de indivíduos de várias idades é justificada pelo fato de obter uma amostra mais heterogênea e representativa, mas por outro lado, as diferenças de composição corporal, ocorridas em função da idade, podem contribuir para um maior erro de estimativa da gordura corporal. Portanto, além de avaliar o tipo de população utilizada na validação, a magnitude do erro padrão deve ser observada ao optar pelo uso dessas equações.

As características étnicas de uma população são outro fator que pode explicar diferenças quanto à composição corporal.

Existem, na literatura, numerosos estudos buscando avaliar essas diferenças e a validade das equações disponíveis em diferentes etnias. A maioria desses estudos tem demonstrado que mulheres negras apresentam maior percentual de gordura corporal, em relação às de etnia branca, que os homens negros possuem maior densidade corporal $^{12,15}$ e que as equações de Durnin \& Wormersley ${ }^{7}$ e de Jackson et al. ${ }^{9}$ apresentam menor acurácia quando aplicadas à população negra ${ }^{15-17}$.

Alguns estudos não encontraram diferenças nos valores de percentual de gordura corporal, mas demonstraram que existem diferenças quanto à localização da gordura. Mulheres brancas apresentaram maior área de tecido adiposo intra-abdominal do que negras ${ }^{18}$. Outros resultados contraditórios têm sido encontrados, como no estudo de Jones et al. ${ }^{19}$, em que mulheres de etnia negra possuíam maior quantidade de massa esquelética do que as de etnia branca de similar idade, peso e estatura. Ressalta-se que resultados diferentes são decorrentes da utilização de métodos de avaliação da composição corporal e de protocolos diferentes.

Diante dos estudos encontrados na literatura está bem documentado que o padrão de composição corporal difere entre as etnias e que as equações existentes de dobras cutâneas, quando aplicadas na população negra, resultam em estimativas de gordura corporal com erros sistemáticos, portanto com baixa acurácia20,21. Além disso, é importante ressaltar que as equações utilizadas para converter a densidade corporal em valores percentuais de gordura corporal foram derivadas de populações de etnia branca, justificando, portanto, a realização de estudos com o objetivo de estabelecer equações específicas para essa população. 


\section{Quantidade de gordura corporal}

Além da etnia e idade, a quantidade de gordura corporal é outro fator que pode implicar em erros sistemáticos na estimativa do percentual de gordura corporal ${ }^{22,23}$. Diante do fato de que as equações existentes foram derivadas de amostras incluindo indivíduos eutróficos e com moderado excesso de peso, estudos buscando avaliar a acurácia dessas equações em indivíduos com sobrepeso e obesidade, têm sido realizados.

Estudo realizado no Brasil $^{22}$ determinou a aplicabilidade e a acurácia da equação de Jackson et al. ${ }^{9}$ em predizer o percentual de gordura corporal em 44 mulheres com idade entre 20 e 40 anos. A média do índice de massa corporal (IMC) na amostra foi de 29,39 $\pm 5,52 \mathrm{~kg} / \mathrm{m}^{2}$. Medida de absorção do raios $X$ de dupla energia (DEXA) foi utilizada como método de referência. Os resultados mostraram que o erro padrão foi aceitável, tanto para a soma de três quanto para a de sete dobras cutâneas, não excedendo 3,5\%. Apesar disso, as equações subestimaram o percentual de gordura corporal comparado aos valores obtidos pelo DEXA. Os autores concluíram que essas equações seriam válidas na avaliação de mulheres eutróficas com percentual de gordura inferior a 30,0\%.

Teran et al. ${ }^{23}$ propõem, para mulheres obesas, equações específicas capazes de predizer com maior acurácia o percentual de gordura corporal.

\section{Nível de atividade física}

O nível de atividade física de um indivíduo influencia diretamente seu perfil de composição corporal. A prática de exercícios físicos é capaz de reduzir a quantidade de gordura corporal e aumentar ou preservar a massa livre de gordura, sendo a magnitude desses efeitos afetada diretamente pela intensidade do exercício ${ }^{24}$.

Quantidades reduzidas de gordura, achado comum em indivíduos com alto nível de atividade física, podem ser estimadas erroneamente, principalmente quando equações generalizadas são utilizadas. A necessidade de equações para grupos populacionais específicos tem sido discutida, e a validade das equações existentes tem sido estudada.

Estudos mostram que a equação de Durnin $\&$ Wormersley ${ }^{7}$ tende a superestimar o percentual de gordura corporal e que a equação de Jackson \& Pollock ${ }^{8}$, que utiliza a soma de três dobras cutâneas, seria a mais adequada para a avaliação de atletas $^{25}$.

No Brasil, Silva et al. ${ }^{26}$ avaliaram a composição corporal de 23 culturistas com idade entre 20 e 56 anos. Os resultados do estudo demonstraram que, apesar da ampla faixa etária da amostra, houve pequena variabilidade nas medidas de dobras cutâneas e perímetros. A média de percentual de gordura corporal encontrada foi de $9,65 \% \pm 0,51$, mostrando a baixa quantidade de gordura nesse grupo.

\section{Desenvolvimento e Validação de Equações no Brasil}

Geralmente as equações são utilizadas na avaliação de indivíduos ou grupos populacionais, sem a realização de validação prévia na amostra que se propõe avaliar. Esse procedimento pode implicar em erros sistemáticos e levar à obtenção de estimativas menos confiáveis. Portanto, verificar a validade das equações para a população que se pretende avaliar é de extrema importância para a obtenção de estimativas mais confiáveis.

Alguns estudos brasileiros têm verificado a validade dessas equações em grupos específicos da população e os resultados mostram que as estimativas da quantidade de gordura corporal, obtidas pela grande maioria das equações disponíveis, diferem muito dos valores encontrados pelo método da pesagem hidrostática ${ }^{27,28}$. Esse fato poderia ser esperado, já que a composição corporal difere segundo uma série de fatores, como discutido anteriormente. Melhores estimativas podem ser alcançadas quando são utilizadas 
equações validadas em população semelhante àquela que se quer avaliar. No entanto, uma validação prévia é recomendada para a aplicação dessas equações.

Estudo realizado na cidade de Santos descreveu o comportamento da quantidade e distribuição da gordura corporal, de acordo com o sexo e idade, por meio de curvas de percentis. A partir das análises realizadas foram propostos, como faixa normal de gordura corporal, os valores de percentis dispostos entre 25 e 75. Indivíduos classificados entre o P75 e o P90, seriam alvo de intervenções dietéticas e/ou prática de atividade física para redução da gordura corporal e aqueles acima do P90 necessitariam de avaliação mais detalhada, para verificar a presença de possíveis fatores de risco associados à obesidade ${ }^{3}$.

Outros pesquisadores têm se empenhado no desenvolvimento e validação, tanto de equações específicas quanto generalizadas, em grupos da população brasileiraa ${ }^{29,30}$.

Salem et al. ${ }^{29}$ estudaram a composição corporal de mulheres militares do Exército Brasileiro com idade entre 18 e 45 anos. Utilizando a pesagem hidrostática como método de referência, foram desenvolvidas e validadas 10 equações a partir de medidas antropométricas, que apresentaram valores de $R$ entre 0,595 e 0,804. Os autores recomendam novos estudos para a validação dessas equações em outros grupos de mulheres brasileiras.

Diante da escassez, no Brasil, de estudos de validação das equações utilizadas para estimar a gordura corporal, a aplicabilidade dessas fórmulas é questionável, portanto mais estudos são necessários para assegurar uma avaliação da composição corporal mais fidedigna.

Devido à enorme variedade de etnias no nosso país e ao crescente aumento da obesidade e da população idosa, as estimativas obtidas na prática clínica e em estudos populacionais, por meio das equações existentes na literatura, podem apresentar erros sistemáticos importantes, influenciando a precisão do diagnóstico. Aspectos como calibração e precisão dos equipamentos utilizados, padronização das técnicas e grau de treinamento do avaliador, devem sempre ser definidos cuidadosamente nos estudos, a fim de minimizar os erros de medidas.

\section{Pesagem Hidrostática: "Padrão-Ouro" para a Validação de Equações?}

A pesagem hidrostática é o método freqüentemente mais utilizado nos estudos de validação de métodos da composição corporal $^{31-34}$, entretanto críticas têm sido feitas quanto ao seu uso ${ }^{33}$. Entre as principais limitações desse método, o uso do modelo de dois compartimentos corporais tem sido a mais citada.

Nesse modelo assume-se que as densidades da massa magra e massa gorda seriam constantes em todos os indivíduos. Portanto, as quantidades de água corporal, massa protéica e óssea não sofreriam alterações segundo o sexo, etnia, idade e nível de atividade física ${ }^{7}$. Como visto anteriormente, todos esses fatores contribuem para diferenças importantes no padrão de composição corporal e, dessa maneira, deveriam ser levados em consideração. Além disso, os procedimentos exigidos para a realização desse método são complexos e exigem laboratório e equipamentos específicos para a avaliação ${ }^{6}$.

Diante do exposto acima, outros métodos vêm sendo utilizados para avaliar a composição corporal e para testar a validade das equações usadas nas estimativas da densidade corporal, como é o caso do uso de isótopos marcados, DEXA e impedância bioelétrica ${ }^{35,36}$. Os resultados desses estudos mostram, em sua maioria, que a utilização das equações na avaliação da composição corporal implica em estimativas errôneas ${ }^{31}$ e apresenta baixa sensibilidade e especificidade ${ }^{37}$.

Outros estudos, além de questionarem o método de pesagem hidrostática, questionam também a análise de somente dois compartimentos corporais e propõem novas equações utilizando medidas de dobras cutâneas para 
predizer a gordura corporal, baseadas no modelo de quatro compartimentos corporais.

Os percentuais de gordura corporal estimado por essas novas equações foram bem próximos aos observados com a equação de quatro compartimentos corporais em ambos os sexos, com diferença média de 0,15\%. Já as equações de Durnin \& Wormersley ${ }^{7}$ e de Jackson \& Pollock ${ }^{8}$, subestimaram o percentual de gordura, obtido com o método de referência, em 2,40\% a $6,60 \%$. Portanto, as novas equações podem predizer com melhor acurácia a gordura corporal nessa população ${ }^{38}$.

\section{O N CLUS Ã O}

Analisando os dados encontrados na literatura, e diante da importância da composição corporal sobre os aspectos de saúde dos indivíduos, é necessário que os profissionais de saúde disponham de métodos confiáveis e de fácil utilização para sua avaliação. Conhecer a validade desses métodos é imprescindível para a aplicação dos mesmos, com objetivo de obter estimativas mais precisas da gordura corporal.

No Brasil, percebe-se uma escassez de estudos nesse sentido e a aplicação de equações de forma indiscriminada na população. Diante da grande variabilidade dos compartimentos corporais entre as populações, é provável que as estimativas da gordura corporal, obtidas com as equações disponíveis, sejam distorcidas dos valores reais.

Verifica-se, portanto, a necessidade de pesquisas, com o objetivo de avaliar a validade das fórmulas utilizadas na população brasileira e, se constatada a inadequação das mesmas, novas equações deverão ser propostas.

\section{REFER Ê N C I A S}

1. Walton C, Lees B, Crook D, Godsland IF, Stevenson JC. Relationships between insulin metabolism, serum lipid profile, body fat distribution and blood pressure in healthy men. Atherosclerosis. 1995; 118(1):35-43.
2. von Eyben FE, Mouritsen E, Holm J, Montvilas P, Dimcevski $G$, et al. Intra-abdominal obesity and metabolic risk factors: a study of young adults. Int J Obes. 2003; 27(8):941-9.

3. Costa RF. Composição corporal: teoria e prática da avaliação. São Paulo: Manole; 2001.

4. Brodie D, Moscrip V, Hutcheon R. Body composition measurement: a review of hydrodensitometry, antropometry, and impedance methods. Nutrition. 1998; 14(3):296-310.

5. Lukaski HC. Methods for the assessment of human body composition: traditional and new. Am J Clin Nutr. 1987; 46(4):537-56.

6. Heyward V. ASEP methods recommendation: body composition assessment. J Exerc Physiol. 2001; 4(4):1-12

7. Durnin JV, Womersley J. Body fat assessed from total body density and its estimation from skinfold thickness: measurements on 481 men and women aged from 16 to 72 years. Br J Nutr. 1974; 32(1): 77-97.

8. Jackson AS, Pollock ML. Generalized equations for predicting body density of men. Br J Nutr. 1978; 40(3):497-504.

9. Jackson AS, Pollock ML, Ward ANN. Generalized equations for predicting body density of women. Med Sci Sports Exerc. 1980; 12(3):175-82.

10. Durnin JV, Rahaman MM. The assessment of the amount of fat in the human body from measurements of skinfold thickness. Br J Nutr. 1967; 21(3):681-9.

11. Bemben MG, Massey BH, Bemben DA, Boileau RA, Misner JE. Age-related patterns in body composition for men aged 20-79 yr. Med Sci Sports Exerc. 1995; 27(2):264-9.

12. Zhu S, Wang Z, Shen W, Heymsfield SB, Heshka S. Percentage body fat ranges associated with metabolic syndrome risk: results based on the third National Health and Nutrition Examination Survey (1988-1994). Am J Clin Nutr. 2003; 78(2):228-35.

13. Martins AL, Memedes MM, Oliveira MPP, Guimarães JNF, Oliveira FP. Análise da composição corporal e do índice de massa corporal de indivíduos de 18 a 50 anos. Cad Saúde Coletiva. 2001; 9(2):97-110.

14. Kyle UG, Genton L, Slosman DO, Pichard C. Fat-free and fat mass percentiles in 5225 healthy subjects aged 15 to 98 years. Nutrition. 2001; 17(7-8):534-41.

15. Jackson AS, Stanforth PR, Gagnon V, Rankinen T, Leon AS, Rao DC, et al. The effect of sex, age and race on estimating percentage body fat from body mass index: the Heritage Family Study. Int J Obes. 2002; 26(6):789-96. 
16. Brandon LJ. Comparison of existing skinfold equations for estimating body fat in African American and white women. Am J Clin Nutr. 1998; 67(6):155-61.

17. Irwin ML, Ainsworth BE, Stolarczyk LM, Heyward $\mathrm{VH}$. Predictive accuracy of skinfold equations for estimating body density of African-American women. Med Sci Sports Exerc. 1998; 30(11): 1654-8.

18. Weinsier RL, Hunter GR, Gower BA, Schutz Y, Darnell BE, Zuckerman PA. Body fat distribution in white and black women: different patterns of intraabdominal and subcutaneous abdominal adipose tissue utilization with weight loss. Am J Clin Nutr. 2001; 74(5):631-6.

19. Jones A Jr, Shen W, St-Onge MP, Gallagher D, Heshka S, Wang Z, et al. Body-composition differences between African American and white women: relation to resting energy requirements. Am J Clin Nutr. 2004; 79(5):780-6.

20. Wagner DR, Heyward VH. Measures of body composition in blacks and whites: a comparative rewiew. Am J Clin Nutr. 2000; 71(6):1392-402.

21. Thomas KT, Keller CS, Holbert KE. Ethnic and age trends for body composition in women residing in the U.S. Southwest: II. total fat. Med Sci Sports Exer. 1997; 29(1):90-8.

22. Bottaro MF, Heyward VH, Bezerra RFA, Wagner DR. Skinfold method vs dual-energy x-ray absorptiometry to assess body composition in normal and obese women. J Exerc Physiol. 2002; 5(2):11-8.

23. Teran JC, Sparks KE, Quinn LM, Fernandez BS, Krey SH, Steffee WP. Percent body fat in obese white females predicted by anthropometric measurements. Am J Clin Nutr. 1991; 53(1):53-7.

24. Fernandez AC, Mello MT, Tufik S, Castro PM, Fisberg M. Influência do treinamento aeróbico e anaeróbico na massa de gordura corporal de adolescentes obesos. Rev Bras Med Esp. 2004; 10(3):152-8.

25. Sinning WE, Dolny DG, Little KD, Cunningham LN, Racaniello A, Siconolfi SF, et al. Validity of "generalized" equations for body composition analysis in male athletes. Med Sci Sports Exerc. 1985; 17(1):124-30.

26. Silva PRP, Trindade RS, De Rose EH. Composição corporal, somatotipo e proporcionalidade de culturistas de elite do Brasil. Rev Bras Med Esp. 2003; 9(6):403-7.

27. Glaner MF, Rodriguez-Añez CR. Validação de equações para estimar a densidade corporal e/ou percentual de gordura para militares masculinos. Treinamento Desportivo. 1999; 4(1):29-36.
28. Guedes DP. Gordura corporal: validação da equação proposta por Faulkner em jovens pertencentes à população brasileira. Artus. 1986; 17:10-3.

29. Salem M, Filho JF, Pires-Neto CS. Desenvolvimento e validação de equações antropométricas específicas para a determinação da densidade corporal de mulheres militares do Exército Brasileiro. Rev Bras Med Esp. 2004; 10(3): 141-6.

30. Petroski EL. Desenvolvimento e validação de equações generalizadas para a estimativa da densidade corporal em adultos [tese]. Santa Maria: UFSM; 1995.

31. Fogelholm M, van Lichtenbelt W. Comparison of body composition methods: a literature analysis. Eur J Clin Nutr. 1997; 51(8):495-503.

32. Stout JR, Eckerson JM, Housh TJ, Johnson GO, Betts NM. Validity of percent body fat estimation in males. Med Sci Sports Exer. 1994; 26(5):632-6.

33. Wang J, Deurenberg P. The validity of predicted body composition in Chinese adults from anthropometry and bioelectrical impedance in comparison with densitometry. Br J Nutr. 1996; 76(2):175-82.

34. Deurenberg P, Deurenberg-Yap M, Wang J, Lin FP, Schmidt G. Prediction of percentage body fat from anthropometry and bioelectrical in Singaporean and Beijing Chinese. Asia Pacific J Clin Nutr. 2000; 9(2):93-8.

35. Clasey JL, Kanaley JÁ, Wideman L, Heymsfield SB, Jeates CD, Gutgesell ME, et al. Validity of methods of body composition assessment in young and older men and women. J Appl Physiol. 1999; 86(5):1728-38.

36. Yao M, Roberts SB, Ma G, Pan H, McCrory MA. Field methods for body composition assessment are valid in healthy Chinese adults. J Nutr. 2002; 132(2):310-7.

37. Friedl KE, Westphal KA, Marchitelli LJ, Patton JF, Chumlea WC, Guo SS. Evaluation of anthropometric equations to assess bodycomposition changes in young women. Am J Clin Nutr. 2001; 73(2):268-75.

38. Peterson MJ, Czerwinski AS, Siervogel RM. Development and validation of skinfold-thickness prediction equations with a 4-compartment model. Am J Clin Nutr. 2003; 77(5):1186-91.

Recebido em: 16/11/2004

Versão final reapresentada em: 25/4/2005 Aprovado em: 30/5/2005 